\title{
Research Article \\ Some Fixed Point Results for Perov-Ćirić-Prešić Type F-Contractions with Application
}

\author{
Abdolsattar Gholidahneh, ${ }^{1}$ Shaban Sedghi $\mathbb{D}^{1},{ }^{1}$ and Vahid Parvaneh $\mathbb{D}^{2}$ \\ ${ }^{1}$ Department of Mathematics, Qaemshahr Branch, Islamic Azad University, Qaemshahr, Iran \\ ${ }^{2}$ Department of Mathematics, Gilan-E-Gharb Branch, Islamic Azad University, Gilan Gharb, Iran \\ Correspondence should be addressed to Shaban Sedghi; sedghi.gh@qaemiau.ac.ir
}

Received 23 March 2020; Revised 27 June 2020; Accepted 28 July 2020; Published 28 August 2020

Academic Editor: Syed Abdul Mohiuddine

Copyright (C) 2020 Abdolsattar Gholidahneh et al. This is an open access article distributed under the Creative Commons Attribution License, which permits unrestricted use, distribution, and reproduction in any medium, provided the original work is properly cited.

Ćirić and Prešić developed the concept of Prešić contraction to Ćirić-Prešić type contractive mappings in the background of a metric space. On the other hand, Altun and Olgun introduced Perov type F-contractions. In this paper, we extend the concept of Ćirić-Prešić contractions to Perov-Ćirić-Prešić type F-contractions. Our results modify some known ones in the literature. To support our main result, an example and an application to nonlinear operator systems are presented.

\section{Introduction}

The Banach contraction principle (BCP) [1] is one of the powerful results in nonlinear analysis. It has many applications in the background of ODE and PDE.

Theorem 1 [1]. Let $(\Delta, d)$ be a complete metric space and let $r: \Delta \longrightarrow \Delta$ so that

$$
d\left(\Upsilon_{\iota,} \Upsilon_{\kappa}\right) \leq \gamma d(\iota, \kappa) \text { for all } \iota, \kappa \in \Delta
$$

where $\gamma \in[0,1)$. Then, there is a unique $\sigma$ in $\Delta$ such that $\sigma=\Upsilon_{\sigma}$. Also, for each $\zeta_{0} \in \Delta$, the sequence $\zeta_{n+1}=\Upsilon \zeta_{n}$ converges to $\sigma$.

The BCP has been extended and generalized in many directions (see [2-4]).

Prešić [5] gave the following result.

Theorem 2 [5]. Let $(\Delta, d)$ be a complete metric space and let $r: \Delta^{k} \longrightarrow \Delta$ ( $k$ is a positive integer). Suppose that

$$
d\left(\Upsilon\left(\zeta_{1}, \cdots, \zeta_{k}\right), \Upsilon\left(\zeta_{2}, \cdots, \zeta_{k+1}\right)\right) \leq \sum_{i=1}^{k} \lambda_{i} d\left(\zeta_{i}, \zeta_{i+1}\right)
$$

for all $\zeta_{1}, \cdots, \zeta_{k+1}$ in $\Delta$, where $\lambda_{i} \geq 0$ and $\sum_{i=1}^{k} \lambda_{i} \in[0,1)$. Then $\gamma$ has a unique fixed point $\zeta^{*}$ (that is $\gamma\left(\zeta^{*}, \ldots, \zeta^{*}\right)=\zeta^{*}$ ). Moreover, for all arbitrary points $\zeta_{1}, \cdots, \zeta_{k+1}$ in $\Delta$, the sequence $\left\{\zeta_{n}\right\}$ defined by $\zeta_{n+k}=\Upsilon\left(\zeta_{n}, \zeta_{n+1}, \cdots, \zeta_{n+k-1}\right)$, converges to $\zeta^{*}$.

It is obvious that for $k=1$, Theorem 2 coincides with the BCP.

Theorem 2 was generalized by Ćirić and Prešić [6] as follows.

Theorem 3 [6]. Let $(\Delta, d)$ be a complete metric space and $r: \Delta^{k} \longrightarrow \Delta$ ( $k$ is a positive integer). Suppose that

$d\left(\Upsilon\left(\zeta_{1}, \cdots, \zeta_{k}\right), \Upsilon\left(\zeta_{2}, \cdots, \zeta_{k+1}\right)\right) \leq \lambda \max \left\{d\left(\zeta_{i}, \zeta_{i+1}\right): 1 \leq i \leq k\right\}$,

for all $\zeta_{1}, \cdots, \zeta_{k+1}$ in $\Delta$, where $\lambda \in[0,1)$. Then $Y$ has a fixed point $\zeta^{*} \in \Delta$. Also, for all points $\zeta_{1}, \cdots, \zeta_{k+1} \in \Delta$, the sequence $\left\{\zeta_{n}\right\}$ defined by $\zeta_{n+k}=\gamma\left(\zeta_{n}, \zeta_{n+1}, \cdots, \zeta_{n+k-1}\right)$, converges to $\zeta^{*}$. The fixed point of $\gamma$ is unique if 


$$
d(\Upsilon(\rho, \cdots, \rho), \Upsilon(\rho, \cdots, \rho))<d(\rho, \rho)
$$

for all $\rho, \varrho \in \Delta$ with $\rho \neq \varrho$.

For more details on Prešić type contractions, we refer the reader to $[2,5,7-11]$.

In this paper, $\mathbb{R}_{+}=[0, \infty), \mathbb{R}^{m}$ denotes the set of $m \times 1$ real matrices, $\mathbb{R}_{+}^{m}$ will be the set of $m \times 1$ real matrices with elements in $[0, \infty), \theta$ denotes the zero $m \times 1$ matrix, $M_{m, m}\left(\mathbb{R}_{+}\right)$denotes the set of all $m \times m$ matrices with elements in $[0, \infty)$, and $\Theta$ will be the zero $m \times m$ matrix, by $I$ the identity $m \times m$ matrix. If $A \in M_{m, m}\left(\mathbb{R}_{+}\right)$, then $A^{T}$ states the transpose matrix of $A$. Let $\pi=\left(\pi_{i}\right)_{i=1}^{m}, \Phi=$ $\left(\omega_{i}\right)_{i=1}^{m} \in \mathbb{R}^{m}$, then by $\pi \preceq \omega$ (resp. $\pi \prec \omega$ ), we suppose $\pi_{i} \leq$ $\omega_{i}\left(\right.$ resp. $\pi_{i}<\omega_{i}$ ) for each $i \in\{1,2, \cdots, m\}$. Also, $\pi \preceq \omega$ and $\omega \geq \pi$ will mean the same.

Let $\Delta$ be a nonempty set and let $V: \Delta \times \Delta \longrightarrow \mathbb{R}^{m}$ be a function. $V$ is called a vector-valued metric, and $(\Delta, V)$ is called a vector-valued metric space, if

(1) $V(\iota, \kappa)=\theta$ if and only if $\iota=\kappa$,

(2) $V(\iota, \kappa)=V(\kappa, \iota)$,

(3) $V(\iota, \kappa) \preceq(\iota, \sigma)+V(\sigma, \kappa)$,

for all $\iota, \kappa, \sigma \in \Delta$.

Example 1 (Example 1.3. of [12]). Let $D_{1}, D_{2}, \cdots, D_{n}$ be usual metrics on $X$.

Then, the mapping $d: X \times X \longrightarrow \mathscr{R}^{n}$ defined by $d(x$, $y)=\left(D_{1}(x, y), D_{2}(x, y), \cdots, D_{n}(x, y)\right)$ is a VVM on $X$.

From now on, we apply VVMS instead of a vector-valued metric space.

The concepts of convergence, Cauchyness, and completeness in a VVMS will be similar as in a usual metric case. Perov [13] stated the contraction mapping principle in the setting of VVMSs. Before stating this theorem, we must remember the following facts:

Let $A \in M_{m, m}\left(\mathbb{R}_{+}\right)$. Then $A$ is said to converge to zero if and only if $A^{n} \longrightarrow \Theta$ as $n \longrightarrow \infty$ (see [14]).

Perov [13] proved the following interesting extension of BCP (see more results in [15-20]).

Theorem 4 [13]. Let $(\Delta, V)$ be a VVMS and $\mathcal{Y}: \Delta \longrightarrow \Delta$ be a mapping such that there exists a matrix $A \in M_{m, m}\left(\mathbb{R}_{+}\right)$such that

$$
V\left(\Upsilon_{\iota}, \gamma_{\kappa}\right) V A V(\iota, \kappa)
$$

for all $\iota, \kappa \in \Delta$. If $A$ is convergent to zero, then

(1) $Y$ has a unique fixed point $\sigma$ in $\Delta$

(2) for all $x_{0} \in \Delta$, the sequence $\left\{x_{n}\right\}$ defined by $x_{n}=Y^{n} x_{0}$ is convergent to $\sigma$

(3) $V\left(x_{n}, \sigma\right) V A^{n}(I-A)^{-1} V\left(x_{0}, r x_{0}\right)$.
In this paper, considering the recent approach of Wardowski [21], we present a generalization of Perov fixed point theorem and Ćirić-Prešić fixed point theorem. Some generalization of Wardowski results can be found in $[22,23]$.

As in [24], let $F: \mathbb{R}_{+}^{m} \longrightarrow \mathbb{R}^{m}$ be a function. Let

(F1) $F$ be strictly increasing in each variable, i.e., $\pi \prec \omega$; then, $F(\pi) \prec F(\omega)$, for all $\pi=\left(\pi_{i}\right)_{i=1}^{m}$ and $\Phi=$ $\left(\omega_{i}\right)_{i=1}^{m} \in \mathbb{R}_{+}^{m}$,

(F2) For each sequence $\left\{\pi_{n}\right\}=\left(\pi_{n}^{(1)}, \pi_{n}^{(2)}, \cdots, \pi_{n}^{(m)}\right)$ of $\mathbb{R}_{+}^{m}$

$\lim _{n \rightarrow \infty} \pi_{n}^{(i)}=0$ if and only if $\lim _{n \rightarrow \infty} \omega_{n}^{(i)}=-\infty$,

for each $i \in\{1,2, \cdots m\}$, where

$$
F\left(\left(\pi_{n}^{(1)}, \pi_{n}^{(2)}, \cdots, \pi_{n}^{(m)}\right)\right)=\left(\omega_{n}^{(1)}, \omega_{n}^{(2)}, \cdots, \omega_{n}^{(m)}\right) .
$$

(F3) There exists $k \in(0,1)$ such that $\lim _{\pi_{i} \rightarrow 0^{+}} \pi_{i}^{k}{\omega_{i}}_{i} 0$ for each $i \in\{1,2, \cdots m\}$, where

$$
F\left(\left(\pi_{1}, \pi_{2}, \cdots, \pi_{m}\right)\right)=\left(\omega_{1}, \omega_{2}, \cdots, \omega_{m}\right)
$$

We denote by $\mathscr{F}^{m}$ the set of all functions $F$ satisfying $(F 1)-(F 3)$

Example 2 [24]. Define $F: \mathbb{R}_{+}^{m} \longrightarrow \mathbb{R}^{m}$ by

$$
F\left(\left(\pi_{1}, \pi_{2}, \cdots, \pi_{m}\right)\right)=\left(\ln \pi_{1}, \ln \pi_{2}, \cdots, \ln \pi_{m}\right),
$$

then $F \in \mathscr{F}^{m}$.

Note that we can define $G: \mathbb{R}^{m} \longrightarrow \mathbb{R}_{+}^{m}$ by

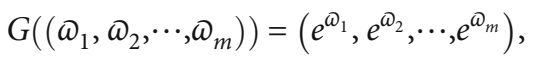

which we can treat it as the inverse of multivariable function $F$.

Note that from now on, $F$ is a continuously differentiable function from all open sets of $A \subseteq \mathbb{R}^{n} \longrightarrow \mathbb{R}^{n}$, and the Jacobian determinant of $F$ at every $p \in A \subseteq \mathbb{R}^{n}$ is nonzero; then, according to inverse function theorem, $F$ is invertible near $p$.

Example 3 [24]. Define $F: \mathbb{R}_{+}^{2} \longrightarrow \mathbb{R}^{2}$ by

$$
F\left(\left(\pi_{1}, \pi_{2}\right)\right)=\left(\ln \pi_{1}, \pi_{2}+\ln \pi_{2}\right),
$$

then $F \in \mathscr{F}^{2}$. 
Example 4 [24]. Define $F: \mathbb{R}_{+}^{3} \longrightarrow \mathbb{R}^{3}$ by

$$
F\left(\left(\pi_{1}, \pi_{2}, \pi_{3}\right)\right)=\left(\ln \pi_{1}, \pi_{2}+\ln \pi_{2},-\frac{1+\pi_{1}}{\sqrt{\pi_{3}}}\right),
$$

then $F \in \mathscr{F}^{3}$.

Considering the class $\mathscr{F}^{m}$, Altun and Olgun [24] introduced the concept of Perov type F-contraction as follows:

Definition 5 [24]. Let $(\Delta, V)$ be a VVMS and $\gamma: \Delta \longrightarrow \Delta$ be a map. If there exist $F \in \mathscr{F}^{m}$ and $\varsigma=\left(\varsigma_{i}\right)_{i=1}^{m} \in \mathbb{R}_{+}^{m}$ such that

$$
\varsigma+F\left(V\left(\Upsilon_{\iota}, \Upsilon_{y}\right)\right) V F(V(\iota, \kappa))
$$

for all $\iota, \kappa \in \Delta$ with $V\left(Y_{\iota}, \Upsilon_{\kappa}\right)>\theta$, then $\Upsilon$ is called a Perov type $F$-contraction.

If we consider $F: \mathbb{R}_{+}^{m} \longrightarrow \mathbb{R}^{m}$ by

$$
F\left(\left(\pi_{1}, \pi_{2}, \cdots, \pi_{m}\right)\right)=\left(\ln \pi_{1}, \ln \pi_{2}, \cdots, \ln \pi_{m}\right),
$$

then (13) turns to Perov contraction [24].

We can present new type contractions in a VVMS, via considering some function $F \in \mathscr{F}^{m}$ in (13).

Theorem 6 [24]. Let $(\Delta, V)$ be a complete VVMS and let $\gamma: \Delta \longrightarrow \Delta$ be a Perov type F-contraction. Then $\mathcal{Y}$ admits a unique fixed point.

In this paper, we introduce the concept of Perov-CirićPrešić type F-contractions. An illustrative example and an application are given to support our main result.

\section{Main Results}

In this section, combining the ideas of Perov, Wardowski, and Ćirić-Prešić, we obtain a new extension of BCP.

Our main result is as follows:

Theorem 7. Let $(\Delta, V)$ be a complete VVMS and let $\Upsilon: \Delta^{k}$ $\longrightarrow \Delta$ ( $k$ is a positive integer). Assume that there exist $F \in$ $\mathscr{F}^{m}$ and $\varsigma=\left(\varsigma_{i}\right)_{i=1}^{m} \in \mathbb{R}_{+}^{m}$ satisfying

$$
\begin{aligned}
\varsigma+ & F\left(V\left(\Upsilon\left(\zeta_{1}, \cdots, \zeta_{k}\right), \Upsilon\left(\zeta_{2}, \cdots, \zeta_{k+1}\right)\right)\right) \\
& \leq F\left(\sup \left\{V\left(\zeta_{1}, \zeta_{2}\right), V\left(\zeta_{2}, \zeta_{3}\right), \cdots, V\left(\zeta_{k}, \zeta_{k+1}\right)\right\}\right),
\end{aligned}
$$

for all $\zeta_{1}, \cdots, \zeta_{k+1} \in \Delta$ with $V\left(\mathcal{Y}\left(\zeta_{1}, \cdots, \zeta_{k}\right), \Upsilon\left(\zeta_{2}, \cdots, \zeta_{k+1}\right)\right)$ $>\theta$. Moreover, let there exists a sequence $\left\{\zeta_{n}\right\}$ in $\Delta$ such that $\zeta_{n+k}=\Upsilon\left(\zeta_{n}, \zeta_{n+1}, \cdots, \zeta_{n+k-1}\right)$ and $V\left(\zeta_{n+k}, \zeta_{n+k+1}\right)>\theta$, for all $n \in \mathbb{N}$. Also, if $\zeta_{n} \longrightarrow v$, then $V\left(\zeta_{n}, v\right)>\theta$, for all $n \in \mathbb{N}$. Then, the sequence $\left\{\zeta_{n}\right\}$ converges to a fixed point of $Y$. Moreover, if for all $\rho, \varrho \in \Delta$ with $\rho \neq \mathrm{\varrho}$,

$$
V(\Upsilon(\rho, \cdots, \rho), \Upsilon(\rho, \cdots, \rho))<V(\rho, \rho)
$$

then the fixed point of $\mathcal{Y}$ is unique.
Proof. For any $n \in \mathbb{N}$, we have

$$
\begin{aligned}
F & \left(V\left(\zeta_{n+k}, \zeta_{n+k+1}\right)\right) \\
& =F\left(V\left(\Upsilon\left(\zeta_{n}, \cdots, \zeta_{n+k-1}\right), \Upsilon\left(\zeta_{n+1}, \cdots, \zeta_{n+k}\right)\right)\right) \\
& \leq F\left(\sup \left\{V\left(\zeta_{n}, \zeta_{n+1}\right), V\left(\zeta_{n+1}, \zeta_{n+2}\right), \cdots, V\left(\zeta_{n+k-1}, \zeta_{n+k}\right)\right\}\right)-\varsigma
\end{aligned}
$$

Therefore,

$$
\begin{aligned}
F( & \left.V\left(\zeta_{k+1}, \zeta_{k+2}\right)\right) \\
& =F\left(V\left(\Upsilon\left(\zeta_{1}, \cdots, \zeta_{k}\right), \Upsilon\left(\zeta_{2}, \cdots, \zeta_{k+1}\right)\right)\right) \\
& \leq F\left(\sup \left\{V\left(\zeta_{1}, \zeta_{2}\right), V\left(\zeta_{2}, \zeta_{3}\right), \cdots, V\left(\zeta_{k}, \zeta_{k+1}\right)\right\}\right)-\varsigma \\
& =F(\Lambda)-\varsigma,
\end{aligned}
$$

where $\Lambda=\sup \left\{V\left(\zeta_{1}, \zeta_{2}\right), V\left(\zeta_{2}, \zeta_{3}\right), \cdots, V\left(\zeta_{k}, \zeta_{k+1}\right)\right\}$. Now,

$$
\begin{aligned}
F( & \left.V\left(\zeta_{k+2}, \zeta_{k+3}\right)\right) \\
& =F\left(V\left(\Upsilon\left(\zeta_{2}, \cdots, \zeta_{k+1}\right), \Upsilon\left(\zeta_{3}, \cdots, \zeta_{k+2}\right)\right)\right) \\
& \leq F\left(\sup \left\{V\left(\zeta_{2}, \zeta_{3}\right), V\left(\zeta_{3}, \zeta_{4}\right), \cdots, V\left(\zeta_{k+1}, \zeta_{k+2}\right)\right\}\right)-\varsigma \\
& \leq F\left(\max \left\{\Lambda, F^{-1}(F(\Lambda)-\varsigma)\right\}\right)-\varsigma=F(\Lambda)-\varsigma .
\end{aligned}
$$

Continuing this approach, we have

$$
\begin{aligned}
F( & \left.V\left(\zeta_{2 k}, \zeta_{2 k+1}\right)\right) \\
& =F\left(V\left(\Upsilon\left(\zeta_{k}, \cdots, \zeta_{2 k-1}\right), \Upsilon\left(\zeta_{k+1}, \cdots, \zeta_{2 k}\right)\right)\right) \\
& \leq F\left(\sup \left\{V\left(\zeta_{k}, \zeta_{k+1}\right), V\left(\zeta_{k+1}, \zeta_{k+2}\right), \cdots, V\left(\zeta_{2 k-1}, \zeta_{2 k}\right)\right\}\right)-\varsigma \\
& \leq F\left(\max \left\{\Lambda, F^{-1}(F(\Lambda)-\varsigma)\right\}\right)-\varsigma=F(\Lambda)-\varsigma, F\left(V\left(\zeta_{2 k+1}, \zeta_{2 k+2}\right)\right) \\
& =F\left(V\left(\Upsilon\left(\zeta_{k+1}, \cdots, \zeta_{2 k}\right), \Upsilon\left(\zeta_{k+2}, \cdots, \zeta_{2 k+1}\right)\right)\right) \\
& \leq F\left(\sup \left\{V\left(\zeta_{k+1}, \zeta_{k+2}\right), V\left(\zeta_{k+2}, \zeta_{k+3}\right), \cdots, V\left(\zeta_{2 k}, \zeta_{2 k+1}\right)\right\}\right)-\varsigma \\
& \leq F\left(F^{-1}(F(\Lambda)-\varsigma)\right)-\varsigma=F(\Lambda)-2 \varsigma, F\left(V\left(\zeta_{3 k}, \zeta_{3 k+1}\right)\right) \\
& =F\left(V\left(\Upsilon\left(\zeta_{2 k}, \cdots, \zeta_{3 k-1}\right), \Upsilon\left(\zeta_{2 k+1}, \cdots, \zeta_{3 k}\right)\right)\right) \\
& \leq F\left(\sup \left\{V\left(\zeta_{2 k}, \zeta_{2 k+1}\right), V\left(\zeta_{2 k+1}, \zeta_{2 k+2}\right), \cdots, V\left(\zeta_{3 k-1}, \zeta_{3 k}\right)\right\}\right)-\varsigma \\
& \leq F\left(\max \left\{F^{-1}(F(\Lambda)-\varsigma), F^{-1}(F(\Lambda)-2 \varsigma)\right\}\right)-\varsigma \\
& =F(\Lambda)-2 \varsigma, F\left(V\left(\zeta_{3 k+1}, \zeta_{3 k+2}\right)\right) \\
& =F\left(V\left(\Upsilon\left(\zeta_{2 k+1}, \cdots, \zeta_{3 k}\right), \Upsilon\left(\zeta_{2 k+2}, \cdots, \zeta_{3 k+1}\right)\right)\right) \\
& \leq F\left(\sup \left\{V\left(\zeta_{2 k+1}, \zeta_{2 k+2}\right), V\left(\zeta_{2 k+2}, \zeta_{2 k+3}\right), \cdots, V\left(\zeta_{3 k}, \zeta_{3 k+1}\right)\right\}\right)-\varsigma \\
& \leq F\left(F^{-1}(F(\Lambda)-2 \varsigma)\right)-\varsigma=F(\Lambda)-3 \varsigma .
\end{aligned}
$$

Continuing this process, we get

$F\left(V\left(\zeta_{p k+i}, \zeta_{p k+i+1}\right)\right) \leq F(\Lambda)-p \varsigma$, for all $p \in V$ and $i \in\{1,2, \cdots, k\}$

Now, taking $V\left(\zeta_{n}, \zeta_{n+1}\right)=\left(\pi_{n}^{1}, \pi_{n}^{2}, \cdots, \pi_{n}^{m}\right)$ and $F\left(\pi_{n}^{1}\right.$, $\left.\pi_{n}^{2}, \cdots, \pi_{n}^{m}\right)=\left(\omega_{n}^{1}, \omega_{n}^{2}, \cdots,{\omega_{n}^{m}}^{m}\right)$, we obtain that 


$$
\begin{aligned}
\left(\omega_{p k+i}^{1}, \omega_{p k+i}^{2}, \cdots, \omega_{p k+i}^{m}\right) & =F\left(\pi_{p k+i}^{1}, \pi_{p k+i}^{2}, \cdots, \pi_{p k+i}^{m}\right) \leq F(\Lambda)-p \varsigma \\
& =\left(r_{1}-p \varsigma_{1}, r_{2}-p \varsigma_{2}, \cdots, r_{m}-p \varsigma_{m}\right),
\end{aligned}
$$

where $\Lambda=\left(\Lambda_{i}\right)_{i=1}^{m}$ and $F\left(\left(\Lambda_{i}\right)_{i=1}^{m}\right)=\left(r_{i}\right)_{i=1}^{m}$. Therefore,

$$
{\omega_{p k+i}^{j}}^{j} \leq r_{j}-p \varsigma_{j} \text { for all } j \in\{1,2, \cdots, m\} .
$$

Passing to the limit, we get $\lim _{p \rightarrow \infty} \omega_{p k+i}^{j}=-\infty$. Therefore, $\lim _{p \rightarrow \infty} \pi_{p k+i}^{j}=0$ for all $j \in\{1,2, \cdots, m\}$. Thus, $\lim _{p \rightarrow \infty} V\left(\zeta_{p k+i}, \zeta_{p k+i+1}\right)=\theta$. From (F3), there exists $\lambda \in(0$, 1) such that

$$
\lim _{p \rightarrow \infty}\left[\pi_{p k+i}^{j}\right]^{\lambda} \omega_{p k+i}^{j}=0 \text {, for all } j \in\{1,2, \cdots, m\} .
$$

From (23),

$$
\left[\pi_{p k+i}^{j}\right]^{\lambda} \Phi_{p k+i}^{j} \leq\left[\pi_{p k+i}^{j}\right]^{\lambda} r_{j}-p\left[\pi_{p k+i}^{j}\right]^{\lambda} \varsigma_{j} \text { for all } j \in\{1,2, \cdots, m\} .
$$

Therefore,

$$
\left[\pi_{p k+i}^{j}\right]^{\lambda} \omega_{p k+i}^{j}-\left[\pi_{p k+i}^{j}\right]^{\lambda} r_{j} \leq-p\left[\pi_{p k+i}^{j}\right]^{\lambda} \varsigma_{j} \leq 0 \text { for all } j \in\{1,2, \cdots, m\} .
$$

Thus, $\lim _{p \rightarrow \infty} p\left[\pi_{p k+i}^{j}\right]^{\lambda}=0$ for all $j \in\{1,2, \cdots, m\}$. So, for any $j \in\{1,2, \cdots, m\}$, there exists $p_{j} \in \mathbb{N}$ such that $p\left[\pi_{p k+i}^{j}\right]^{\lambda} \leq$ 1 , for all $p \geq p_{j}$. Thus, $\pi_{p k+i}^{j} \leq 1 / p^{1 / \lambda}$, for all $p \geq p_{j}$. Putting $p_{0}=\max \left\{p_{j}: 1 \leq j \leq m\right\}$, we have $\pi_{p k+i}^{j} \leq 1 / p^{1 / \lambda}$ for all $p \geq$ $p_{0}$ and all $i \in\{1,2, \cdots, k\}$. We claim that $\left\{\zeta_{n}\right\}$ is a Cauchy sequence. Consider two elements $m, n \in \mathbb{N}$ so that $p_{0} k \leq$ $n<m$. Then, there are $p, q \in \mathbb{N}$ and $i, j \in\{1,2, \cdots, k\}$ such that $p_{0} \leq p \leq q, n=p k+i$, and $m=q k+j$. Now, we have

$$
\begin{aligned}
V\left(\zeta_{n}, \zeta_{m}\right) & =V\left(\zeta_{p k+i}, \zeta_{q k+j}\right) \leq \sum_{r=p}^{q} \sum_{l=1}^{k} V\left(\zeta_{r k+l}, \zeta_{r k+l+1}\right) \\
& \leq \sum_{r=p}^{q} k\left(\frac{1}{r^{1 / \lambda}}, \cdots, \frac{1}{r^{1 / \lambda}}\right) \leq k\left(\sum_{r=p}^{q} \frac{1}{r^{1 / \lambda}}, \cdots, \sum_{r=p}^{q} \frac{1}{r^{1 / \lambda}}\right)
\end{aligned}
$$

As $n, m \longrightarrow \infty$, we have $p, q \longrightarrow \infty$. Thus, the last term in (27) converges to $\theta$, and so $\left\{\zeta_{n}\right\}$ is a Cauchy sequence in $(\Delta, V)$. Since $(\Delta, V)$ is a complete VVMS, there is $v \in \Delta$ so that $\lim _{n \rightarrow \infty} \zeta_{n}=v$. Now, we shall prove that $v$ is a fixed point of $r$. To see this, we have

$$
\begin{aligned}
& V\left(\zeta_{n+k}, \Upsilon(v, \cdots, v)\right) \\
&= V\left(\Upsilon\left(\zeta_{n}, \zeta_{n+1} \cdots, \zeta_{n+k-1}\right), \Upsilon(v, \cdots, v)\right) \\
& \leq V\left(\Upsilon\left(\zeta_{n}, \zeta_{n+1}, \cdots, \zeta_{n+k-1}\right), \Upsilon\left(\zeta_{n+1}, \zeta_{n+2}, \cdots, \zeta_{n+k-1}, v\right)\right) \\
&+V\left(\Upsilon\left(\zeta_{n+1}, \zeta_{n+2}, \cdots, \zeta_{n+k-1}, v\right), \Upsilon\left(\zeta_{n+2}, \zeta_{n+3}, \cdots, \zeta_{n+k-1}, v, v\right)\right) \\
&+\cdots+V\left(\Upsilon\left(\zeta_{n+k-1}, v, \cdots, v\right), \Upsilon(v, v, \cdots, v)\right) \\
& \leq F^{-1}\left[F\left(\max \left\{V\left(\zeta_{n}, \zeta_{n+1}\right), \cdots, V\left(\zeta_{n+k-2}, \zeta_{n+k-1}\right), V\left(\zeta_{n+k-1}, v\right)\right\}\right)-\varsigma\right] \\
&+F^{-1}\left[F \left(\operatorname { m a x } \left\{V\left(\zeta_{n+1}, \zeta_{n+2}\right), \cdots, V\left(\zeta_{n+k-2}, \zeta_{n+k-1}\right),\right.\right.\right. \\
&\left.\left.\left.\times V\left(\zeta_{n+k-1}, v\right)\right\}\right)-\varsigma\right] \cdots+F^{-1}\left[F\left(V\left(\zeta_{n+k-1}, v\right)\right)-\varsigma\right] \longrightarrow \theta,
\end{aligned}
$$

as $n \longrightarrow \infty$. Thus,

$$
V(v, \Upsilon(v, \cdots, v))=\lim _{n \rightarrow \infty} V\left(\zeta_{n+k}, \Upsilon(v, \cdots, v)\right)=\theta
$$

Therefore, $v=r(v, \cdots, v)$. Suppose that $u, v$ are two distinct fixed points of $r$. From our hypothesis,

$$
V(u, v)=V(\Upsilon(u, \cdots, u), \Upsilon(v, \cdots, v)))<V(u, v),
$$

which is a contradiction. Thus, the fixed point of $r$ is unique.

Note that by taking

$$
F\left(\left(\pi_{1}, \pi_{2}, \cdots, \pi_{m}\right)\right)=\left(\ln \pi_{1}, \ln \pi_{2}, \cdots, \ln \pi_{m}\right),
$$

the above theorem reduces to the following theorem.

Theorem 8. Let $(\Delta, V)$ be a complete VVMS and $r: \Delta^{k}$ $\longrightarrow \Delta$ ( $k$ is a positive integer). Suppose that there exist $F \in$ $\mathscr{F}^{m}$ and $\varsigma=\left(\varsigma_{i}\right)_{i=1}^{m} \in \mathbb{R}_{+}^{m}$ satisfying

$V\left(\Upsilon\left(\zeta_{1}, \cdots, \zeta_{k}\right), \Upsilon\left(\zeta_{2}, \cdots, \zeta_{k+1}\right)\right) \leq A \sup \left\{V\left(\zeta_{i}, \zeta_{i+1}\right): i=1, \cdots, k\right\}$,

where

$$
A=\left(\begin{array}{cccc}
e^{-\varsigma_{1}} & 0 & \cdots & 0 \\
0 & e^{-\varsigma_{2}} & \cdots & 0 \\
\vdots & \vdots & \ddots & \vdots \\
0 & 0 & \cdots & e^{-\varsigma_{m}}
\end{array}\right)_{m \times m}
$$

Let the sequence $\left\{\zeta_{n}\right\}$ in $\Delta$ be such that $\zeta_{n+k}=\gamma\left(\zeta_{n}\right.$, $\left.\zeta_{n+1}, \cdots, \zeta_{n+k-1}\right)$ and $V\left(\zeta_{n+k}, \zeta_{n+k+1}\right)>\theta$, for all $n \in \mathbb{N}$. Also, 
if $\zeta_{n} \longrightarrow v$, then $V\left(\zeta_{n}, v\right)>\theta$, for all $n \in \mathbb{N}$. Then, the sequence $\left\{\zeta_{n}\right\}$ converges to a fixed point of $\mathcal{Y}$. Also, if

$$
V(\mathcal{Y}(\rho, \cdots, \rho), \mathcal{Y}(\rho, \cdots, \rho))<V(\rho, \rho)
$$

for all $\rho, \varrho \in \Delta$ with $\rho \neq \mathrm{Q}$, then the fixed point of $Y$ is unique.

We present an example to support our main result.

Example 5. Let $\Delta=\left\{\varsigma_{n}=1 / n^{2}: n=1,2, \cdots\right\} \cup\left\{\varsigma_{0}=0\right\}, V(\rho$, $\mathrm{Q})=(|\rho-\mathrm{Q}|,|\rho-\mathrm{Q}|)$, and define $\mathcal{Y}: \Delta^{2} \longrightarrow \Delta$ by

$$
\Upsilon\left(\varsigma_{n}, \varsigma_{m}\right)=\left(\begin{array}{ll}
\varsigma_{\max \{m, n\}+1}, & n, m \geq 1, \\
0, & n=0 \text { or } m=0 .
\end{array}\right.
$$

Firstly, note that for all $m, n \in \mathbb{N} \cup\{0\}$ with $m \neq n$, from Example 2.3 of [25], we have

$$
\begin{array}{r}
\left|\varsigma_{n+1}-\varsigma_{m+1}\right|^{1 / \sqrt{\left|\varsigma_{n+1}-\zeta_{m+1}\right|}}\left|\varsigma_{n}-\varsigma_{m}\right|^{-1 / \sqrt{\left|\varsigma_{n}-\zeta_{m}\right|}} \leq \frac{1}{2}, \\
\frac{1}{\sqrt{\left|\varsigma_{n+1}-\varsigma_{m+1}\right|}}-\frac{1}{\sqrt{\left|\varsigma_{n}-\varsigma_{m}\right|}} \geq 1 .
\end{array}
$$
$\mathbb{N}$ and

As we know, $\varsigma_{n+2}=\min \left\{\varsigma_{n+1}, \varsigma_{n+2}\right\}=\mathcal{Y}\left(\varsigma_{n}, \varsigma_{n+1}\right)$, for all

$$
\begin{aligned}
V\left(\varsigma_{n+2}, \varsigma_{n+3}\right) & =\left(\left|\varsigma_{n+2}-\varsigma_{n+3}\right|,\left|\varsigma_{n+2}-\varsigma_{n+3}\right|\right) \\
& =\left(\left|\frac{1}{(n+2)^{2}}-\frac{1}{(n+3)^{2}}\right|,\left|\frac{1}{(n+2)^{2}}-\frac{1}{(n+3)^{2}}\right|\right) \\
& >(0,0)=\theta .
\end{aligned}
$$

Also, $\varsigma_{n}=1 / n^{2} \longrightarrow 0$ and

$$
V\left(\varsigma_{n}, 0\right)=\left(\left|\varsigma_{n}-0\right|,\left|\varsigma_{n}-0\right|\right)=\left(\frac{1}{n^{2}}, \frac{1}{n^{2}}\right) \succ(0,0)=\theta .
$$

Define $F: \mathbb{R}_{+}^{2} \longrightarrow \mathbb{R}^{2}$ by

$$
F\left(\left(\pi_{1}, \pi_{2}\right)\right)= \begin{cases}\left(\frac{\ln \pi_{1}}{\sqrt{\pi_{1}}}, \frac{-1}{\sqrt{\pi_{2}}}\right), \quad \pi_{1} \leq e \\ \left(\frac{\pi_{1}}{e \sqrt{e}}, \frac{-1}{\sqrt{\pi_{2}}}\right), \quad \pi_{1}>e .\end{cases}
$$

Obviously, $F \in \mathscr{F}^{2}$. Also, take $\varsigma=\left(\varsigma_{1}, \varsigma_{2}\right)=(\ln 2,1)$. We have

$$
\begin{aligned}
& \varsigma+F(V(\Upsilon(\varepsilon, \varepsilon), \Upsilon(\varepsilon, \sigma))) \\
& \leq F(\max \{V(\varepsilon, \varepsilon), V(\varepsilon, \sigma)\}) \\
& \Leftrightarrow(\ln 2,1)+F(|\Upsilon(\varepsilon, \varepsilon)-\Upsilon(\varepsilon, \sigma)|,|\Upsilon(\varepsilon, \varepsilon)-\Upsilon(\varepsilon, \sigma)|) \\
& \leq F(\max \{|\varepsilon-\varepsilon|,|\varepsilon-\sigma|\}) \Leftrightarrow(\ln 2,1) \\
& +\left(\frac{\ln (|\Upsilon(\varepsilon, \varepsilon)-\Upsilon(\varepsilon, \sigma)|)}{\sqrt{|\Upsilon(\varepsilon, \varepsilon)-\Upsilon(\varepsilon, \sigma)|}}, \frac{-1}{\sqrt{|\Upsilon(\varepsilon, \varepsilon)-\Upsilon(\varepsilon, \sigma)|}}\right) \\
& \leq\left(\frac{\ln (\max \{|\varepsilon-\varepsilon|,|\varepsilon-\sigma|\})}{\sqrt{\max \{|\varepsilon-\varepsilon|,|\varepsilon-\sigma|\}}}, \frac{-1}{\sqrt{\max \{|\varepsilon-\varepsilon|,|\varepsilon-\sigma|\}}}\right) \\
& \Leftrightarrow \ln 2+\frac{\ln (|\Upsilon(\varepsilon, \varepsilon)-\Upsilon(\varepsilon, \sigma)|)}{\sqrt{|\Upsilon(\varepsilon, \varepsilon)-\Upsilon(\varepsilon, \sigma)|}} \leq \frac{\ln (\max \{|\varepsilon-\varepsilon|,|\varepsilon-\sigma|\})}{\sqrt{\max \{|\varepsilon-\varepsilon|,|\varepsilon-\sigma|\}}}, 1 \\
& +\frac{-1}{\sqrt{|\Upsilon(\varepsilon, \varepsilon)-Y(\varepsilon, \sigma)|}} \leq \frac{-1}{\sqrt{\max \{|\varepsilon-\varepsilon|,|\varepsilon-\sigma|\}}}
\end{aligned}
$$

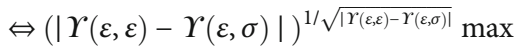

$$
\begin{aligned}
& \times\{|\varepsilon-\varepsilon|,|\varepsilon-\sigma|\}^{-1 / \sqrt{\max \{|\varepsilon-\varepsilon|,|\varepsilon-\sigma|\}}} \\
& \leq \frac{1}{2}, \frac{1}{\sqrt{|\Upsilon(\varepsilon, \varepsilon)-\Upsilon(\varepsilon, \sigma)|}}-\frac{1}{\sqrt{\max \{|\varepsilon-\varepsilon|,|\varepsilon-\sigma|\}}} \geq 1,
\end{aligned}
$$

for any $\varepsilon, \varepsilon, \sigma \in \Delta$. Now, Let $\varepsilon=\varsigma_{n}, \varepsilon=\varsigma_{m}$, and $\sigma=\varsigma_{p}$. If $m$ $\geq \max \{n, p\}$, then

$$
\begin{aligned}
V(\mathcal{Y}(\varepsilon, \varepsilon), \mathcal{Y}(\varepsilon, \sigma)) & =V\left(\mathcal{Y}\left(\varsigma_{n}, \varsigma_{m}\right), \mathcal{Y}\left(\varsigma_{m}, \varsigma_{p}\right)\right) \\
& =V\left(\varsigma_{m+1}, \varsigma_{m+1}\right)=(0,0)=\theta
\end{aligned}
$$

So, we may assume that either $m<n$ or $m<p$. We consider the following cases:

Case 1. $n \leq m<p$. Let $n=0$. If $n=m=0$, then

$$
V(\Upsilon(\varepsilon, \varepsilon), \Upsilon(\varepsilon, \sigma))=(0,0)=\theta
$$

If $0=n<m$, then

$$
\begin{aligned}
& (|\Upsilon(\varepsilon, \varepsilon)-\Upsilon(\varepsilon, \sigma)|)^{1 / \sqrt{|Y(\varepsilon, \varepsilon)-Y(\varepsilon, \sigma)|}} \\
& \cdot \max \{|\varepsilon-\varepsilon|,|\varepsilon-\sigma|\}^{1 / \sqrt{\max \{|\varepsilon-\varepsilon|,|\varepsilon-\sigma|\}}} \\
& =\left(\varsigma_{p+1}\right)^{1 / \sqrt{\varsigma_{p+1}}}\left(\varsigma_{m}\right)^{1 / \sqrt{\varsigma_{m}}}=\left(\frac{1}{(p+1)^{2}}\right)^{p+1}\left(\frac{1}{m^{2}}\right)^{-m} \\
& \leq\left(\frac{1}{(p+1)^{2}}\right)^{p+1}\left(\frac{1}{p^{2}}\right)^{-p} \leq \frac{1}{(p+1)^{2}} \\
& \leq \frac{1}{2} \frac{1}{\sqrt{|\Upsilon(\varepsilon, \varepsilon)-\Upsilon(\varepsilon, \sigma)|}}-\frac{1}{\sqrt{\max \{|\varepsilon-\varepsilon|,|\varepsilon-\sigma|\}}} \\
& =\frac{1}{\sqrt{\varsigma_{p+1}}}-\frac{1}{\sqrt{\varsigma_{m}}}=p+1-m \geq p+1-p=1 \text {, }
\end{aligned}
$$


and if $n>0$, then

$$
\begin{aligned}
& (|\Upsilon(\varepsilon, \varepsilon)-\Upsilon(\varepsilon, \sigma)|)^{1 / \sqrt{|Y(\varepsilon, \varepsilon)-Y(\varepsilon, \sigma)|}} \max \\
& \cdot\{|\varepsilon-\varepsilon|,|\varepsilon-\sigma|\}^{-1 / \sqrt{\max \{|\varepsilon-\varepsilon|,|\varepsilon-\sigma|\}}} \\
& \leq\left(\varsigma_{m+1}-\varsigma_{p+1}\right)^{1 / \sqrt{\varsigma_{m+1}-\varsigma_{p+1}}}\left(\varsigma_{m}-\varsigma_{p}\right)^{-1 / \sqrt{\varsigma_{m}-\varsigma_{p}}} \\
& \leq \frac{1}{2} \frac{1}{\sqrt{|\Upsilon(\varepsilon, \varepsilon)-Y(\varepsilon, \sigma)|}}-\frac{1}{\sqrt{\max \{|\varepsilon-\varepsilon|,|\varepsilon-\sigma|\}}} \\
& =\frac{1}{\sqrt{\varsigma_{p+1}}}-\frac{1}{\sqrt{\varsigma_{m}}}=p+1-m \geq p+1-p=1 \text {. }
\end{aligned}
$$

Case 2. $m<p \leq n$. Here, if $m=0$, then

$$
\begin{aligned}
& (|\Upsilon(\varepsilon, \varepsilon)-Y(\varepsilon, \sigma)|)^{1 / \sqrt{|\Upsilon(\varepsilon, \varepsilon)-Y(\varepsilon, \sigma)|}} \max \{|\varepsilon-\varepsilon|,|\varepsilon-\sigma|\}^{-1 / \sqrt{\max \{|\varepsilon-\varepsilon||\varepsilon-\sigma|\}}} \\
& \quad=\left(\varsigma_{n+1}\right)^{1 / \sqrt{\varsigma_{n+1}}}\left(\varsigma_{n}\right)^{-1 / \sqrt{\varsigma_{n}}}=\left(\frac{1}{(n+1)^{2}}\right)^{n+1}\left(\frac{1}{n^{2}}\right)^{-n} \leq \frac{1}{(n+1)^{2}} \leq \frac{1}{2},
\end{aligned}
$$

and

$$
\begin{gathered}
\frac{1}{\sqrt{|\Upsilon(\varepsilon, \varepsilon)-\Upsilon(\varepsilon, \sigma)|}}-\frac{1}{\sqrt{\max \{|\varepsilon-\varepsilon|,|\varepsilon-\sigma|\}}} \\
=\frac{1}{\sqrt{\varsigma_{n+1}}}-\frac{1}{\sqrt{\varsigma_{n}}}=n+1-n \geq n+1-n=1,
\end{gathered}
$$

and if $m>0$, then

$$
\begin{aligned}
& (|\Upsilon(\varepsilon, \varepsilon)-Y(\varepsilon, \sigma)|)^{1 / \sqrt{|Y(\varepsilon, \varepsilon)-\Upsilon(\varepsilon, \sigma)|}} \\
& \cdot \max \{|\varepsilon-\varepsilon|,|\varepsilon-\sigma|\}^{-1 / \sqrt{\max \{|\varepsilon-\varepsilon|,|\varepsilon-\sigma|\}}} \\
& \leq\left(\varsigma_{n+1}-\varsigma_{p+1}\right)^{1 / \sqrt{\varsigma_{n}-\varsigma_{p}}}\left(\varsigma_{n}-\varsigma_{p}\right)^{-1 / \sqrt{\varsigma_{n}-\varsigma_{p}}} \leq \frac{1}{2} \text {, }
\end{aligned}
$$

and

$$
\begin{aligned}
& \frac{1}{\sqrt{|\Upsilon(\varepsilon, \varepsilon)-\Upsilon(\varepsilon, \sigma)|}}-\frac{1}{\sqrt{\max \{|\varepsilon-\varepsilon|,|\varepsilon-\sigma|\}}} \\
& =\frac{1}{\sqrt{\varsigma_{n+1}-\varsigma_{p+1}}}-\frac{1}{\sqrt{\varsigma_{n}-\varsigma_{p}}} \geq 1 .
\end{aligned}
$$

Case 3. $m \leq n<p$. In this case, if $m=0$, then

$$
\begin{aligned}
& (|Y(\varepsilon, \varepsilon)-Y(\varepsilon, \sigma)|)^{1 / \sqrt{|Y(\varepsilon, \varepsilon)-\Upsilon(\varepsilon, \sigma)|}} \max \\
& \quad \cdot\{|\varepsilon-\varepsilon|,|\varepsilon-\sigma|\}^{-1 / \sqrt{\max \{|\varepsilon-\varepsilon||| \varepsilon-\sigma \mid\}}} \\
& \leq\left(\varsigma_{p+1}\right)^{1 / \sqrt{\varsigma_{p+1}}}\left(\varsigma_{p}\right)^{-1 / \sqrt{\varsigma_{p}}}=\left(\frac{1}{(p+1)^{2}}\right)^{p+1}\left(\frac{1}{p^{2}}\right)^{-p} \\
& \leq \frac{1}{(p+1)^{2}} \leq \frac{1}{2},
\end{aligned}
$$

and

$$
\begin{aligned}
& \frac{1}{\sqrt{|\mathcal{Y}(\varepsilon, \varepsilon)-\Upsilon(\varepsilon, \sigma)|}}-\frac{1}{\sqrt{\max \{|\varepsilon-\varepsilon|,|\varepsilon-\sigma|\}}} \\
& =\frac{1}{\sqrt{\varsigma_{p+1}}}-\frac{1}{\sqrt{\varsigma_{p}}}=p+1-p=1,
\end{aligned}
$$

and if $m>0$, then.

$$
\begin{aligned}
& (|Y(\varepsilon, \varepsilon)-Y(\varepsilon, \sigma)|)^{1 / \sqrt{|Y(\varepsilon, \varepsilon)-\Upsilon(\varepsilon, \sigma)|}} \max \\
& \quad \cdot\{|\varepsilon-\varepsilon|,|\varepsilon-\sigma|\}^{-1 / \sqrt{\max \{|\varepsilon-\varepsilon|,|\varepsilon-\sigma|\}}} \\
& \leq\left(\varsigma_{n+1}-\varsigma_{p+1}\right)^{1 / \sqrt{\varsigma_{n+1}-\zeta_{p+1}}}\left(\varsigma_{n}-\varsigma_{p}\right)^{-1 / \sqrt{\varsigma_{n}-\zeta_{p}}} \leq \frac{1}{2},
\end{aligned}
$$

and

$$
\begin{aligned}
& \frac{1}{\sqrt{|\Upsilon(\varepsilon, \varepsilon)-Y(\varepsilon, \sigma)|}}-\frac{1}{\sqrt{\max \{|\varepsilon-\varepsilon|,|\varepsilon-\sigma|\}}} \\
& =\frac{1}{\sqrt{\zeta_{n+1}-\zeta_{p+1}}}-\frac{1}{\sqrt{\zeta_{n}-\zeta_{p}}} \geq 1 .
\end{aligned}
$$

Case 4. $p \leq m<n$. Here, if $p=0$, then

$$
\begin{aligned}
& (|\Upsilon(\varepsilon, \varepsilon)-Y(\varepsilon, \sigma)|)^{1 / \sqrt{|\gamma(\varepsilon, \varepsilon)-Y(\varepsilon, \sigma)|}} \max \\
& \cdot\{|\varepsilon-\varepsilon|,|\varepsilon-\sigma|\}^{-1 / \sqrt{\max \{|\varepsilon-\varepsilon|,|\varepsilon-\sigma|\}}} \\
& \leq\left(\varsigma_{n+1}\right)^{1 / \sqrt{\varsigma_{n+1}}}\left(\varsigma_{n}\right)^{-1 / \sqrt{\varsigma_{n}}}=\left(\frac{1}{(n+1)^{2}}\right)^{n+1}\left(\frac{1}{n^{2}}\right)^{-n} \\
& \leq \frac{1}{(n+1)^{2}} \leq \frac{1}{2}
\end{aligned}
$$

and

$$
\begin{aligned}
& \frac{1}{\sqrt{|\mathcal{Y}(\varepsilon, \varepsilon)-\Upsilon(\varepsilon, \sigma)|}}-\frac{1}{\sqrt{\max \{|\varepsilon-\varepsilon|,|\varepsilon-\sigma|\}}} \\
& =\frac{1}{\sqrt{\varsigma_{n+1}}}-\frac{1}{\sqrt{\varsigma_{n}}}=n+1-n=1,
\end{aligned}
$$

and if $p>0$, then

$$
\begin{aligned}
& (|\mathcal{Y}(\varepsilon, \varepsilon)-\Upsilon(\varepsilon, \sigma)|)^{1 / \sqrt{|Y(\varepsilon, \varepsilon)-\Upsilon(\varepsilon, \sigma)|}} \max \\
& \quad \cdot\{|\varepsilon-\varepsilon|,|\varepsilon-\sigma|\}^{-1 / \sqrt{\max \{|\varepsilon-\varepsilon|,|\varepsilon-\sigma|\}}} \\
& \leq\left(\varsigma_{n+1}-\varsigma_{m+1}\right)^{1 / \sqrt{\varsigma_{n+1}-\varsigma_{m+1}}}\left(\varsigma_{n}-\varsigma_{m}\right)^{-1 / \sqrt{\varsigma_{n}-\varsigma_{m}}} \leq \frac{1}{2},
\end{aligned}
$$


and

$$
\begin{aligned}
& \frac{1}{\sqrt{|\Upsilon(\varepsilon, \varepsilon)-\Upsilon(\varepsilon, \sigma)|}}-\frac{1}{\sqrt{\max \{|\varepsilon-\varepsilon|,|\varepsilon-\sigma|\}}} \\
& =\frac{1}{\sqrt{\varsigma_{n+1}-\varsigma_{m+1}}}-\frac{1}{\sqrt{\varsigma_{n}-\varsigma_{m}}} \geq 1 .
\end{aligned}
$$

Also, let $\rho, \rho \in \Delta$ with $\rho \neq \rho$. Without loss of any generality, let $\rho=\varsigma_{n}, \rho=\varsigma_{m}$ with $n<m$. If $n=0$, then

$$
\begin{aligned}
V(\Upsilon(\rho, \rho), \Upsilon(\rho, \rho)) & =V\left(\Upsilon\left(\varsigma_{0}, \varsigma_{0}\right), \Upsilon\left(\varsigma_{m}, \varsigma_{m}\right)\right)=V\left(0, \varsigma_{m+1}\right) \\
& =\left(\varsigma_{m+1}, \varsigma_{m+1}\right) \prec\left(\varsigma_{m}, \varsigma_{m}\right)=V\left(0, \varsigma_{m}\right) \\
& =V(\rho, \rho), V\left(\varsigma_{1}, \varsigma_{m-1}\right)=\frac{m(m-1)}{2}-1 \\
& <\frac{m(m+1)}{2}-1=V\left(\varsigma_{1}, \varsigma_{m}\right)=V(\rho, \rho),
\end{aligned}
$$

and if $n>0$, then

$$
\begin{aligned}
V(\Upsilon(\rho, \rho), \Upsilon(\rho, \rho)) & =V\left(\Upsilon\left(\varsigma_{n}, \varsigma_{n}\right), \Upsilon\left(\varsigma_{m}, \varsigma_{m}\right)\right)=V\left(\varsigma_{n+1}, \varsigma_{m+1}\right) \\
& =\left(\left|\varsigma_{n+1}-\varsigma_{m+1}\right|,\left|\varsigma_{n+1}-\varsigma_{m+1}\right|\right) \\
& =\left(\frac{1}{(n+1)^{2}}-\frac{1}{(m+1)^{2}}, \frac{1}{(n+1)^{2}}-\frac{1}{(m+1)^{2}}\right) \\
& <\left(\frac{1}{n^{2}}-\frac{1}{m^{2}}, \frac{1}{n^{2}}-\frac{1}{m^{2}}\right)=V\left(\varsigma_{n}, \varsigma_{m}\right) \\
& =V(\rho, \rho) .
\end{aligned}
$$

We see that all of the conditions of Theorem 2 are satisfied. Thus, $\boldsymbol{Y}$ has a unique fixed point. Here, $\boldsymbol{r}\left(\varsigma_{0}, \varsigma_{0}\right)=\varsigma_{0}$, and $\varsigma_{0}$ is the unique fixed point.

We present an example in an infinite dimensional sequence space $\ell_{1}$ which is adapted from the above example, and so, we leave the details for the reader.

Let $A$ be the space of all convergent sequences $\left(a_{n}\right)$ for which $a_{i}=1 / n^{2}$ ( $n$ is an arbitrary natural number) for exactly one $i$ and $a_{j}=0$ for other indices.

Let $\quad \Delta=A \cup\left\{\varsigma_{0}=(0,0,0, \cdots)\right\}, \quad V\left(\left(a_{n}\right)_{m=1}^{\infty},\left(b_{n}\right)_{n=1}^{\infty}\right)=$ $\left(\sum_{m, n=1}^{\infty}\left|a_{n}-b_{n}\right|, \Sigma_{m, n=1}^{\infty}\left|a_{n}-b_{n}\right|\right)$, and define $r: \Delta^{2} \longrightarrow$ $\Delta$ by

$$
r\left(\left(a_{n}\right)_{n=1}^{\infty},\left(b_{n}\right)_{n=1}^{\infty}\right)=\left(\begin{array}{ll}
\left(0, \cdots, 0, a_{\max \{i, j\}+1}=\frac{1}{n^{2}}, 0, \cdots\right), & \left(a_{n}\right) \neq(0,0,0, \cdots) \text { and }\left(b_{n}\right) \neq(0,0,0, \cdots) \\
(0,0,0, \cdots), & \left(a_{n}\right)=(0,0,0, \cdots) \text { or }\left(b_{n}\right)=(0,0,0, \cdots) .
\end{array}\right.
$$

Define $F: \mathbb{R}_{+}^{2} \longrightarrow \mathbb{R}^{2}$ by

$$
F\left(\left(\pi_{1}, \pi_{2}\right)\right)= \begin{cases}\left(\frac{\ln \pi_{1}}{\sqrt{\pi_{1}}}, \frac{-1}{\sqrt{\pi_{2}}}\right), & \pi_{1} \leq e \\ \left(\frac{\pi_{1}}{e \sqrt{e}}, \frac{-1}{\sqrt{\pi_{2}}}\right), & \pi_{1}>e .\end{cases}
$$

Obviously, $F \in \mathscr{F}^{2}$. Also, take $\varsigma=\left(\varsigma_{1}, \varsigma_{2}\right)=(\ln 2,1)$.

Reviewing the above example, we can show that all of the conditions of Theorem 2 are satisfied. Thus, $r$ has a unique fixed point. Here, $\boldsymbol{Y}((0,0,0, \cdots),(0,0,0, \cdots))=(0,0,0, \cdots)$ and $(0,0,0, \cdots)$ is the unique fixed point.

\section{Application}

Let $\left(E,\|\cdot\|_{E}\right)$ be a Banach space and $A_{1}, \cdots, A_{k}: E^{k} \longrightarrow E$ be $k$ nonlinear operators. In this section, motivated by the work in [26], we will present a result on existence of a solution for the following semilinear operator system:

$$
\begin{gathered}
A_{1}\left(\iota_{1}, \iota_{2}, \cdots, \iota_{k}\right)=\iota_{1}, \\
\vdots \\
A_{k}\left(\iota_{1}, \iota_{2}, \cdots, \iota_{k}\right)=\iota_{k} .
\end{gathered}
$$

Similar systems which appear in various branches of mathematics could be seen in [27].

Let $\Delta=E^{k}$ and define $V: \Delta \times \Delta \longrightarrow \mathbb{R}^{k}$, for $u=\left(\iota_{1}, \cdots, \iota_{k}\right)$, $v=\left(\varepsilon_{1}, \cdots, \varepsilon_{k}\right) \in \Delta$ by $V(u, v)=\left(\left\|\iota_{1}-\varepsilon_{1}\right\|_{E}, \cdots,\left\|\iota_{k}-\varepsilon_{k}\right\|_{E}\right)$. Evidently, $(\Delta, V)$ is a complete VVMS.

If we define a mapping $r: \Delta^{k} \longrightarrow \Delta$ by

$$
r(u, u, \cdots, u)=\left(A_{1}\left(\iota_{1}, \iota_{2}, \cdots, \iota_{k}\right), \cdots, A_{k}\left(\iota_{1}, \iota_{2}, \cdots, \iota_{k}\right)\right),
$$

then the system (61) can be written as a fixed point problem such as

$$
\Upsilon(u, u, \cdots, u)=\left(\iota_{1}, l_{2}, \cdots, \iota_{k}\right)=u,
$$

in the space $\Delta$. Therefore, applying Theorem 2 , we investigate the sufficient hypothesis which leads to the existence of a solution of problem (63).

Theorem 9. Assume that there exist positive real numbers $\varsigma_{i}$ $(i=1, \cdots, k)$ such that

$$
\left\|A_{i}\left(\iota_{1}, \iota_{2}, \cdots, \iota_{k}\right)-A_{i}\left(\varepsilon_{1}, \varepsilon_{2}, \cdots, \varepsilon_{k}\right)\right\|_{E} \leq e^{-\varsigma_{i}}\left\|\iota_{i}-\varepsilon_{i}\right\|_{E},
$$

for all $u=\left(\iota_{1}, \cdots, \iota_{k}\right), v=\left(\varepsilon_{1}, \cdots, \varepsilon_{k}\right) \in E^{k}$ with $\iota_{i} \neq \varepsilon_{i}$. Then, the system (61) has a unique solution in $E^{k}$. 
Proof. By the inequality (64), we have

$$
\left(\varsigma_{i}+\ln \left\|A_{i}\left(\iota_{1}, \iota_{2}, \cdots, \iota_{k}\right)-A_{i}\left(\kappa_{1}, \kappa_{2}, \cdots, \kappa_{k}\right)\right\|_{E}\right) \leq \ln \left\|\iota_{i}-\kappa_{i}\right\|_{E},
$$

for all $i=1, \cdots, k$. Hence, we get

$$
\begin{aligned}
& \left(\varsigma_{1}+\ln \left\|A_{1}\left(u_{1}\right)-A_{1}\left(u_{2}\right)\right\|_{E}, \cdots, \varsigma_{k}+\ln \left\|A_{k}\left(u_{1}\right)-A_{k}\left(u_{2}\right)\right\|_{E}\right) \\
& \quad \preceq\left(\ln \left\|\iota_{1}-\kappa_{1}\right\|_{E}, \cdots, \ln \left\|\iota_{k}-\kappa_{k}\right\|_{E}\right) .
\end{aligned}
$$

Taking the function $F \in \mathscr{F}^{k}$ as $F\left(\pi_{1}, \cdots, \pi_{k}\right)=\left(\ln \pi_{1}, \cdots\right.$, $\ln \pi_{k}$ ), the above inequality can be written as

$$
\begin{aligned}
& \left(\varsigma_{1}, \cdots, \varsigma_{k}\right)+F\left(\left(\left\|A_{1} u_{1}-A_{1} u_{2}\right\|_{E}, \cdots,\left\|A_{k} u_{1}-A_{k} u_{2}\right\|_{E}\right)\right) \\
& \quad \leq F\left(\left(\left\|\iota_{1}-\kappa_{1}\right\|_{E}, \cdots,\left\|\iota_{k}-\kappa_{k}\right\|_{E}\right)\right) \\
& \quad=F\left(V\left(u_{1}, u_{2}\right)\right), V F\left(\sup \left\{V\left(u_{1}, u_{2}\right), V\left(u_{1}, u_{2}\right), \cdots, V\left(u_{1}, u_{2}\right)\right\}\right)
\end{aligned}
$$

or, equivalently,

$$
\begin{aligned}
& \left(\varsigma_{1}, \cdots, \varsigma_{k}\right)+F\left(V\left(\Upsilon\left(u_{1}, \cdots, u_{k}\right), \Upsilon\left(u_{2}, \cdots, u_{k+1}\right)\right)\right) \\
& \quad \leq F\left(\sup \left\{V\left(u_{1}, u_{2}\right), V\left(u_{2}, u_{3}\right), \cdots, V\left(u_{k}, u_{k+1}\right)\right\}\right),
\end{aligned}
$$

where $\varsigma=\left(\varsigma_{1}, \cdots, \varsigma_{k}\right)$. Thus, applying Theorem $2, \gamma$ possesses a unique fixed point in $\Delta=E^{k}$, or, equivalently, the semilinear operator system (61) has a unique solution in $E^{k}$.

\section{Data Availability}

No data were used to support this study.

\section{Conflicts of Interest}

The authors declare that they have no competing interests.

\section{Authors' Contributions}

All authors read and approved the manuscript.

\section{References}

[1] S. Banach, "Sur les opérations dans les ensembles abstraits et leur application aux équations intègrales," Fundamenta Mathematicae, vol. 3, pp. 133-181, 1922.

[2] V. Parvaneh, F. Golkarmanesh, and R. George, "Fixed points of Wardowski-ciric-Presic type contractive mappings in a partial rectangular b-metric space," Journal of Mathematical Analysis, vol. 8, no. 1, pp. 183-201, 2017.

[3] Z. Mustafa, J. R. Roshan, V. Parvaneh, and Z. Kadelburg, "Some common fixed point results in ordered partial bmetric spaces," Journal of inequalities and applications, vol. 2013, no. 1, 2013

[4] J. R. Roshan, V. Parvaneh, Z. Kadelburg, and N. Hussain, "New fixed point results in b-rectangular metric spaces," Nonlinear Analysis: Modelling and Control, vol. 21, no. 5, pp. 614634, 2016.
[5] S. B. Prešić, "Sur une classe d'inèquations aux differences finies et sur la convergence de certaines suites," Publications de l'Institut Mathématique Belgrade, vol. 5, no. 19, pp. 75-78, 1965.

[6] L. B. Ćirić and S. B. Prešić, "On Prešić type generalisation of Banach contraction mapping principle," Acta Mathematica Universitatis Comenianae, vol. LXXVI, no. 2, pp. 143-147, 2007.

[7] Y. Z. Chen, "A Prešić type contractive condition and its applications," Nonlinear Analysis, vol. 71, pp. 2012-2017, 2009.

[8] S. Shukla, N. Mlaiki, and H. Aydi, "On (G, G')-Prešić-Ćirić operators in graphical metric spaces," Mathematics, vol. 7, no. 5, p. 445, 2019.

[9] S. Shukla, D. Gopal, and R. Rodríguez-López, "Fuzzy-PrešicCiric operators and applications to certain nonlinear differential equations," Mathematical Modelling and Analysis, vol. 21, no. 6, pp. 811-835, 2017.

[10] S. Shukla and H. K. Nashine, "Cyclic-Prešić-Ćirić operators in metric- like spaces and fixed point theorems," Nonlinear Analysis-Modelling and Control, vol. 21, no. 2, pp. 261-273, 2016.

[11] S. Shukla, "Set-valued Prešić-Ćirić type contraction in 0complete partial metric spaces," Matematicki Vesnik, vol. 66, no. 2, pp. 178-189, 2014.

[12] S. S. Rao, "On vector valued metric spaces," Palestine Journal of Mathematics, vol. 4, no. 2, pp. 416-418, 2015.

[13] A. I. Perov, "On the Cauchy problem for a system of ordinary differential equations," Pviblizhen. Met. Reshen. Differ. Uvavn., vol. 2, pp. 115-134, 1964.

[14] R. S. Varga, Matrix Iterative Analysis, Springer Series in Computational Mathematics, vol. Volume 27, Springer, Berlin, 2000.

[15] F. Vetro and S. Radenović, "Some results of Perov type in rectangular cone metric spaces," Journal of Fixed Point Theory and Applications, vol. 20, no. 1, 2018.

[16] M. Abbas, V. Rakočević, and A. Iqbal, "Fixed points of Perov type contractive mappings on the set endowed with a graphic structure," Revista de la Real Academia de Ciencias Exactas, Físicas y Naturales. Serie A. Matemáticas, vol. 112, no. 1, pp. 209-228, 2018.

[17] M. Cvetković, "On the equivalence between Perov fixed point theorem and Banach contraction principle," Filomat, vol. 31, no. 11, pp. 3137-3146, 2017.

[18] M. Cvetković and V. Rakočević, "Common fixed point results for mappings of Perov type," Mathematische Nachrichten, vol. 288, no. 16, pp. 1873-1890, 2015.

[19] M. Cvetković and V. Rakočević, "Extensions of Perov theorem," Carpathian Journal of Mathematics, vol. 31, no. 2, pp. 181-188, 2015.

[20] D. Ilić, M. Cvetković, L. Gajić, and V. Rakočević, "Fixed points of sequence of Ćirić generalized contractions of Perov type," Mediterranean Journal of Mathematics, vol. 13, no. 6, pp. 3921-3937, 2016.

[21] D. Wardowski, "Fixed points of a new type of contractive mappings in complete metric spaces," Fixed Point Theory and Applications, vol. 2012, no. 1, 2012.

[22] N. Hussain, G. Ali, I. Iqbal, and B. Samet, "The existence of solutions to nonlinear matrix equations via fixed points of multivalued F-contractions," Mathematics, vol. 8, no. 2, p. 212, 2020.

[23] N. Hussain, A. Latif, I. Iqbal, and M. A. Kutbi, "Fixed point results for multivalued F-contractions with application to 
integral and matrix equations," Journal of Nonlinear and Convex Analysis, vol. 20, no. 11, pp. 2297-2311, 2019.

[24] I. Altun and M. Olgun, "Fixed point results for Perov type Fcontractions and an application," Journal of Fixed Point Theory and Applications, vol. 22, p. 46, 2020.

[25] G. Minak, A. Helvac, and I. Altun, "Ćirić type generalized Fcontractions on complete metric spaces and fixed point results," Filomat, vol. 28, no. 6, pp. 1143-1151, 2014.

[26] I. Altun, N. Hussain, M. Qasim, and H. H. Al-Sulami, "A new fixed point result of Perov type and its application to a semilinear operator system," Mathematics, vol. 7, no. 11, p. 1019, 2019.

[27] R. Precup, "The role of matrices that are convergent to zero in the study of semi-linear operator systems," Mathematical and Computer Modelling, vol. 49, no. 3-4, pp. 703-708, 2009. 